\title{
Identification of Sickle Cell Anemia Using Deep Neural Networks
}

\author{
Sagar Yeruva ${ }^{1 *}$, M. Sharada Varalakshmi ${ }^{2}$, B. Pavan Gowtham ${ }^{1}$, \\ Y. Hari Chandana ${ }^{1}$, PESN. Krishna Prasad ${ }^{3}$ \\ ${ }^{1}$ Department of CSE, VNR Vignana Jyothi Institute of Engineering \& Technology, Hyderabad, Telangana, India \\ ${ }^{2}$ Department of CSE, Methodist College of engineering \& Technology, Hyderabad, Telangana, India \\ ${ }^{3}$ Sri Venkateswara College of Engineering, Tirupati, Andhra Pradesh, India
}

\begin{abstract}
A molecule called hemoglobin is found in red blood cells that holds oxygen all over the body Hemoglobin is elastic, round, and stable in a healthy human. This makes it possible to float across red blood cells. But the composition of hemoglobin is unhealthy if you have sickle cell disease. It refers to compact and bent red blood cells. The odd cells obstruct the flow of blood. It is dangerous and can result in severe discomfort, organ damage, heart strokes, and other symptoms. The human life expectancy can be shortened as well. The early identification of sickle calls will help people recognize signs that can assist antibiotics, supplements, blood transfusion, pain-relieving medications, and treatments etc. The manual assessment, diagnosis, and cell count are time consuming process and may result in misclassification and count since millions of red blood cells are in one spell. When utilizing data mining techniques such as the multilayer perceptron classifier algorithm, sickle cells can be effectively detected with high precision in the human body. The proposed approach tackles the limitations of manual research by implementing a powerful and efficient MLP (Multi-Layer Perceptron) classification algorithm that distinguishes Sickle Cell Anemia (SCA) into three classes: Normal (N), Sickle Cells $(\mathrm{S})$ and Thalassemia $(\mathrm{T})$ in red blood cells. This paper also presents the precision degree of the MLP classifier algorithm with other popular mining and machine learning algorithms on the dataset obtained from the Thalassemia and Sickle Cell Society (TSCS) located in Rajendra Nagar, Hyderabad, Telangana, India.
\end{abstract}

\author{
Keywords: \\ Anemia, Sickle Cell (SC); \\ Sickle Cell Anemia (SCA); \\ Sickle Cell Disease (SCD); \\ MLP Classifier; \\ Thalassemia. \\ Article History: \\ Received: 18 December 2020 \\ Revised: 27 February 2021 \\ Accepted: 08 March 2021 \\ Published: $01 \quad$ April 2021
}

\section{1- Introduction}

Natural blood passes in a tiny circular form that brings oxygen to organs from parts of the human body that are spherical and have a lifespan of around 120 days which produces a new blood cell every 120 days [1]. Sickle cell anemia is a particular form of irregular blood disorder that causes natural hemoglobin in the red blood cells to change its shape. Sickle cell form is Sticky and hard Disc form, which allows the blood supply to cease in the human body [2]. The average cycle of sickle cells is 10 to 20 days. Because of the involvement of sickle cells [3] in hemoglobin, it may cause extreme episodes of discomfort, tissue loss, and serious complications, in some cases leading to death [4].

In above Figure 1, it is clear indicated that the usual blood flow carries oxygen through all areas of the blood without slowing, where the sickle cell form becomes sticky and the blood flow ceases [5] at some point. Since the blood flow

\section{*CONTACT: Sagar_y@vnrvjiet.in}

DOI: http://dx.doi.org/10.28991/esj-2021-01270

(C) 2021 by the authors. Licensee ESJ, Italy. This is an open access article under the terms and conditions of the Creative Commons Attribution (CC-BY) license (https://creativecommons.org/licenses/by/4.0/). 
ceases, it may cause serious body pains and heart strokes etc. Sickle cell was found in the black community and was later seen in citizens of many ethnic groups, including citizens from parts of the Middle East, Central India, the Mediterranean Region, Italy, and Greece in particular [6]. Treatment for Sickle Cell Disease by taking early drugs such as Antibiotics, Blood Transfusion, Bone Marrow. For at least 1-2 months, most physicians prescribe blood transfusion, and patients can take antibiotics to treat risks, including persistent pain. SCD is an uncommon blood condition [7] in the human body that is now found in the hemoglobin of newborn babies. SCD is a crescent-shaped cell that is stiff, sticky, and associates with other cells. If one organ is gradually damaged it can spread across the body, which may contribute to death. To escape this kind of illness, early treatment is needed to stop severe problems (Blood Transfusion). A person develops hemoglobin $\mathrm{S}$, which is triggered by two defective genes.

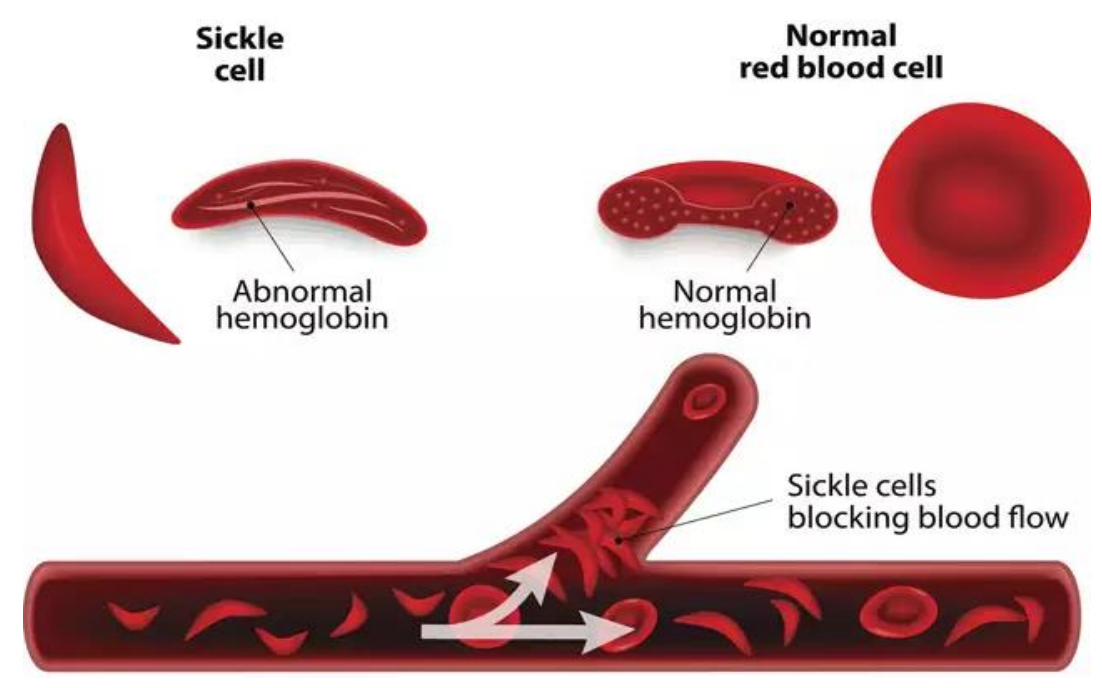

Figure 1. Blood Flow of Sickle Cells.

\section{1-1-Syndromes and Indications of Sickle Cell Anemia [8]}

Signs of sickle cell anemia and symptoms typically begin at the age of 5 months. They differ and alter with time from individual to individual. Symptoms and signs can involve:

1) Episodes of pain: The RBC prevents blood supply into narrow blood vessels joints, abdomen, and lungs which is one of the main signs of sickle cells. Chronic discomfort has been identified in bones, ulcers, and joint injuries in adults and teenagers.

2) Distressing swelling of hands and feet: RBC inhibits the blood supply of hands and feet because of the sickle cell involvement in the body and may swell in the hands and feet.

3) Frequent infections: Due to cellular sickle damage in bodies that trigger organ harm (infection fights i.e. spleen). Physicians also prescribe vaccinations and drugs to avoid diseases that endanger patient health, such as pneumonia.

3) Disrupted development: If RBCs in the human body are decreased by a lack of oxygen, which can contribute to slower development.

4) Vision problems: RBC prevents sickle cell blood flow through tiny blood vessels. Such cells do not supply the eyes with oxygen, which may contribute to retinal injury. It creates trouble with the vision.

\section{1-2-Sickle Cell Complications [9]}

1) Heart Stroke: Cardiac stroke may arise when the blood supply to the brain is disrupted by the sickle cells. The stroke signs include arms and legs fatigue or numbness, frequent speech difficulties, lack of consciousness [10].

2) Acute chest syndrome: It induces thorn discomfort, fever, and breathing trouble. The issue, which can also lead to life-threats, is very severe. Clinical intervention is needed with the usage of antibiotics and other therapies [11].

3) Pulmonary Hypertension: High blood pressure in the lungs (pulmonary hypertension) can occur in patients with sickle cell anemia. This condition affects not only the children but also adults. Symptoms can trigger breathing difficulties and tiredness.

4) Leg Ulcers: SCA (sickle cell anemia) can cause open sores, where ulcers on the legs can develop. 
5) Gallstones. A substance such as bilirubin may be produced by the RBC breakdown. If bilirubin has a high body level that contributes to gallstones.

\section{1-3-Statistical Information [12]}

\section{In Indian Scenario [12]}

1) First portrayed in northern Tamil Nadu in 1952 in the Nilgiri Hills.

2) The sickle cell gene is now considered to be common among citizens in central India's Deccan plateau with less emphasis in northern Kerala and Tamil Nadu.

\section{In worldwide Scenario [12]}

1) Sickle cells were originally unknown, but now spread across the world and are found particularly in the Western Hemisphere (Caribbean, South America, and Central America), Saudi Arabia, Asia, and the Mediterranean countries (Greece, ITA, Turkey), Sub-Saharan Africa[13].

2) In the African-American countries, sickle cell death is more observed; between 1999 and 2002, sickle cell mortality [14] in children younger than 4 years declined by $42 \%$. The reduced sickle cell in 2000 , which defends against infectious diseases of the pneumococcal community, was detected through vaccination.

3) Sickle cell disease was reported in California, Illinois, and New York during the period 1990-1994 by the newborn screening of mortality infants.

4) In Africa-American children with SCD in California and Illinois, mortality rates stood at 1.5 per 100 by the end of 1995. African / American or black babies born in California and Illinois have a mortality rate of 2.0 per 100 Blacks or African-Americans during this time [15].

5) SCD is one of the biggest public health issues, with the total expense of around $\$ 475$ million for Hospitalization related to SCD in the US of 75000.

The rest of this paper is presented as follows: A comprehensive review of the sickle cell crisis and various attempts to find the crises through computational methods is described in section-2 (Literature Review). Complete overview of the dataset used in this experimentation is mentioned in section-3. Section-4 includes the previous results obtained on the dataset and usage of Multi-Layer Perceptron that escalates the prediction accuracy. Section-5 presents the results obtained from Multi-Layer Perceptron and the results comparison with other models can be found.

\section{2- Literature Review}

\section{2-1-Sickle Cell Disease-A Comprehensive Study and Usage of Technology for Diagnosis [16]}

A comprehensive study of sickle cell disease with the properties, symptoms, signs, and treatment procedure is mentioned. The authors also present the comprehensive study and characteristics of the disease with other similar diseases (like thalassemia) along with technological implications and usage in the field of sickle cell disease.

\section{2-2-Healthy and Unhealthy Red Blood Cell Detection in Human Blood Smears using Neural Networks [17]}

The following methods are used in this paper for diagnosing anemia: A hemoglobin level recognition system, focused on a microscopic blood smear examination, and an RBC classification. The author used neural networks to classify and count [18] three forms of anemia (Sickle cells, elliptocytosis cells, microsite cells, and cells with unknown shapes) by utilizing circular Hough transformations and morphological tools in a microscope.

\section{2-3-Emerging Point-of-care Technologies for Sickle Cell Disease Diagnostics [19]}

In this paper, POC platforms are developed to ensure cost efficiency and portability which enables millions of people in low-resource countries to be diagnosed with sickle cell anemia as potentially improbable. A comprehensive literature analysis was conducted to evaluate the sensitivity and specification of several POC diagnostics produced for SCD with an emphasis on resource constraint use. In this paper, Microfluidic paper-based devices, Paper-based tests for screening newborns, Sickle cell detection using a smartphone etc. are discussed.

\section{2-4-Data Mining Technique using WEKA Classification for Sickle Cell Disease [17]}

In this paper, two classification strategies, J48 and Random Tree are used, to forecast cell disease, heavily affected in Gujarat tribal regions, and then contrasted J48 and Random Tree categorization strategies for the mining method. The author contrasted this strategy to the Random Tree. For this prediction method, the WEKA platform is used as an opensource application. 


\section{2-5-A Comparative Analysis by KNN, SVM \& ELM Classification to Detect Sickle Cell Anemia [8]}

In this paper, the dataset of blood samples is taken in picture format. In the image pre-processing phase, gray photography, noise filters, and image enhancement are transformed. This paper also presents Fuzzy C which classifies regular and sickle cells. For analysis, the graphical and mathematical properties are used. The author used KNN, SVM, and Extreme Learning Machine classification tools [20] to evaluate images [21].

\section{2-6-Edge Detection of Sickle Cells in Red Blood Cells [4]}

In this paper, the sickle and the regular cells are identified by measuring the smaller, largest, and medium radius of each cell by contrasting that with the typical cell dimension. For this method, sickle cells with red circles are labelled using edge detection techniques [22]. Regular, sickle, and other irregular cells are located using a microscope in the clinical process, but manually it is impossible to identify superimposed cells. In this automatic process, blood cells overlapped and incomplete are first observed, and then separated to regular or irregular cells by means of specific edge finding algorithms depending on the shape of each cell derived from a microscopic picture [23].

\section{2-7-Automatic Blood Cell Segmentation using K-Mean Clustering from Microscopic Thin Blood Images [25]}

In this source, Savkare [25] presents an automatic blood cell segmentation in parasitic diseases using K-Means algorithms on online image library (http://www.dpd.cdc.gov/dpdx/ and is tested on 60 microscopic thin blood images with an accuracy of $98.89 \%$ for clustering.

\section{2-8-Machine Learning Approaches to the Application of Disease Modifying Therapy for Sickle Cell using Classification Models [26]}

Khalaf [26] has obtained the results with several algorithms on sickle cell disease especially using Elman-Jordan Hybrid Neural Network (EJNN) and Levenberg Marquardt algorithm (LEVNN) where the accuracy is very higher than other models.

\section{2-9-Detection of Anemia Disease in Human Red Blood Cells using Cell Signature, Neural Networks and SVM [22]}

Elsalamony et al. has done the detection of sickle cell anemia disease in RBC using various popular algorithms like cell signature, Support Vector Machine (SVM), Back Propagation (BP) and Self-Organizing Map (SOM) neural networks by testing with 45 microscopic colour images from 15 patients who is already suffering with this kind of anemia.

\section{3- About Dataset}

As part of this project [A collaborative Research project awarded by Jawaharlal Nehru Technological University, Hyderabad, TEQIP-III (funding agency) for Rs.3,00,000/-, we had executed a Memorandum of Understanding (MoU) between our Institute named Vallurupalli Nageswara Rao Vignana Jyothi Institute of Engineering \& Technology, Bachupally, Hyderabad, Telangana, India 500090 (VNRVJIET) and Thalassemia and Sickle Cell Society, Rajendra Nagar, Hyderabad, Telangana, India (TSCS) (MoU dated 15 Oct 2019). The details of TSCS is accessible from https://www.tscsindia.org/.

The MoU aimed with the following objectives:

- Data Sharing and

- Technology Transfer

We have received a dataset of 1387 records of patients who have approached TSCS for the diagnosis purpose. These records are shared to us as part of $\mathrm{MoU}$ and the records are pre-processed to maintain confidentiality, anonymity that meets data privacy of patients. This data of the patients gathered during August 2017 to August 2019.

After pre-processing, 13 attributes are identified along with the class label (Diagnosis of a Blood sample). The complete list of attributes, their meaning and can be found from our previous research article (in press) presented at ICACECS-2020 (International Conference on Advances in Computer Engineering and Communication Systems 2020) and available in Gowtham et al. (2020) [27].

\section{4- Implementation}

In Gowtham et al. (2020) [27] study, we have presented the results of prediction of identification of Thalassemia and Sickle Cell traits in the blood samples using the classification algorithms [28] is summarized [27] in Table 1: 
Table 1. Comparison study of results of various classification algorithms.

\begin{tabular}{cccc}
\hline S. No. & Name of the Classification Algorithm & $\begin{array}{c}\text { Result of prediction } \\
\text { accuracy }(\mathbf{\%})\end{array}$ & $\begin{array}{c}\text { Dataset Size (No of observations } \\
\text { X No of Parameters) }\end{array}$ \\
\hline 1 & Support Vector Machine (SVM) & 76.00 & 1387 X 13 \\
2 & K-Nearest Neighbor Algorithm (KNN) & 77.00 & 1387 X 13 \\
3 & Logistic Regression (L) & 78.00 & 1387 X 13 \\
4 & Decision Tree Classifier (DTC) & 95.00 & 1387 X 13 \\
5 & Random Forest Algorithm (RF) & 96.00 & 1387 X 13 \\
\hline
\end{tabular}

Later, another 100 records of patients were received who approached TSCS (during the period September 2019 to August 2020) that could help us to experiment and test with another model called "Multi-Layer Perceptron (MLP) Classifier".

The aim of this work is the production and identification of sickle cell anemia with the aid of Multilayer Perceptron. The model architecture includes three stages, such as Dataset preparation, Analyzing dataset, splitting dataset (Training and Testing), and usage of classification algorithms [29]. The proposed classifier, named Multi-Layer Perceptron Classifier gives the detection results on the sickle cell anemia. The implementation of the proposed technique in PYTHON. The system is evaluated in terms of Accuracy and Log Loss to show the performance of the technique.

\section{4-1- Dataset Preparation}

As stated above, 1387 patients with 13 parameters are included in the data collection obtained from Thalassemia and the Sickle Cell Society. The parameters are explained in Table 2.

Table 2. The 13 parameters present in the dataset with an explanation.

\begin{tabular}{|c|c|c|}
\hline S. No. & Parameter Name & Description \\
\hline 1 & AGE & The period that person has lived \\
\hline 2 & Hemoglobin (HB) & A red protein responsible for transporting Oxygen in the blood of vertebrates. \\
\hline 3 & Hematocrit (HCT) & The ratio of the volume of red blood cells to the total volume of blood \\
\hline 4 & RBC Distribution Width (RDW) & $\begin{array}{l}\text { It is a measure of the range of variation of red blood cell volume that is reported as part of a } \\
\text { standard complete blood count }\end{array}$ \\
\hline 5 & Mean Corpuscular Volume (MCV) & It is a measure of the average volume of a blood corpuscle. \\
\hline 6 & Mean Corpuscular Hemoglobin (MCH) & It is the average mass of hemoglobin per red blood cell in a sample of blood. \\
\hline 7 & $\begin{array}{l}\text { Mean Corpuscular Hemoglobin } \\
\text { Concentration }(\mathrm{MCHC})\end{array}$ & It is a measure of the concentration of hemoglobin in each volume of packed red blood cells. \\
\hline 8 & Red Blood Cell (RBC) & The blood cells that carry oxygen \\
\hline 9 & Reticulocytes (RETIC) & $\begin{array}{l}\text { An immature red blood cell without a nucleus, having a granular or reticulated appearance } \\
\text { when suitably stained. }\end{array}$ \\
\hline 10 & Fetal Hemoglobin (HBF) & It is the main oxygen carrier protein in the human fetus. \\
\hline 11 & HBA0 & It is defined as the non-glycated hemoglobin. \\
\hline 12 & HBA2 & $\begin{array}{l}\text { It is a normal variant of hemoglobin A that consists of two alpha and two delta chains and are } \\
\text { found at low levels in normal human blood }\end{array}$ \\
\hline 13 & Diagnosis & $\begin{array}{l}\text { Diagnosis is the output variable; We need to predict based on a set of features(inputs) wither } \\
\text { it is a Normal Cell / Sickle Cell / Thalassemia Cell }\end{array}$ \\
\hline
\end{tabular}

\section{4-2-Analyzing Dataset and Splitting Dataset}

Every patient's details (i.e. diagnosis) with AS, AT, BT, EA, EE, ET, NN, SC, SS, ST, TM, TT, have been named by thalassemia society. The 12 diagnosis names are translated into three major classes as mentioned below

1) $\mathrm{NN}$

2) AS, SC, SS, ST

$$
\begin{aligned}
& =\mathrm{N}(\text { Normal Cells }) \\
& =\mathrm{S}(\text { Sickle Cells })
\end{aligned}
$$

Usually datasets are dealt-in machine learning that have multiple labels in one or more columns. The training data is also labelled in words to render it comprehensible or understandable in human terms. 
Label Encoding involves transforming labels into numeric types to make them readable by the computer. Machine learning algorithms will then help to determine how to run the labels. For the organized data set in supervised learning, it is an essential pre-processing phase.

The dataset is split using a train-test-split function which is helpful to partition the data into a training set and test set (of $80 \%$ and $20 \%$ respectively) provided by scikit-learn. The training set includes a known value, and the model learns from that data such that other data can later be generalized. The reference dataset (or subclass) to check the accuracy of our model for this subclass is available.

\section{4-3-Multi-Layer Perceptron (MLP) Classifier}

MLP Classifier is often called a perceptron classifier of multilayer representation [30] that indicates a neural network itself. To conduct the classification process, MLP Classifier depends on an elementary Neural Network. That is ANN (Artificial Neural Network). The expression MLP is used ineffectively, often almost for every forward ANN, and often specifically refers to multi-layer perceptron networks (with threshold activation). Now and then, multilayer perceptrons are called "vanilla" neural networks, particularly if they involve one secret layer. It is rather robust and can usually be used to know the mapping from input to output. The lab findings are long-term avoidance.

MLPs are valuable in analysis to solve problems stochastically, also offering estimated answers for highly complicated problems like an approximation of fitness. MLPs allow effective classification of algorithms as a special case of regressions, where the answer variable is categorical. In the 1980s, MLPs became common for machine learning approaches, and they found implementations in a variety of different fields, such as speech recognition, Imagerecognition, and machine translation tools. Because of the achievements of deep learning, curiosity in history contact networks resurfaced.

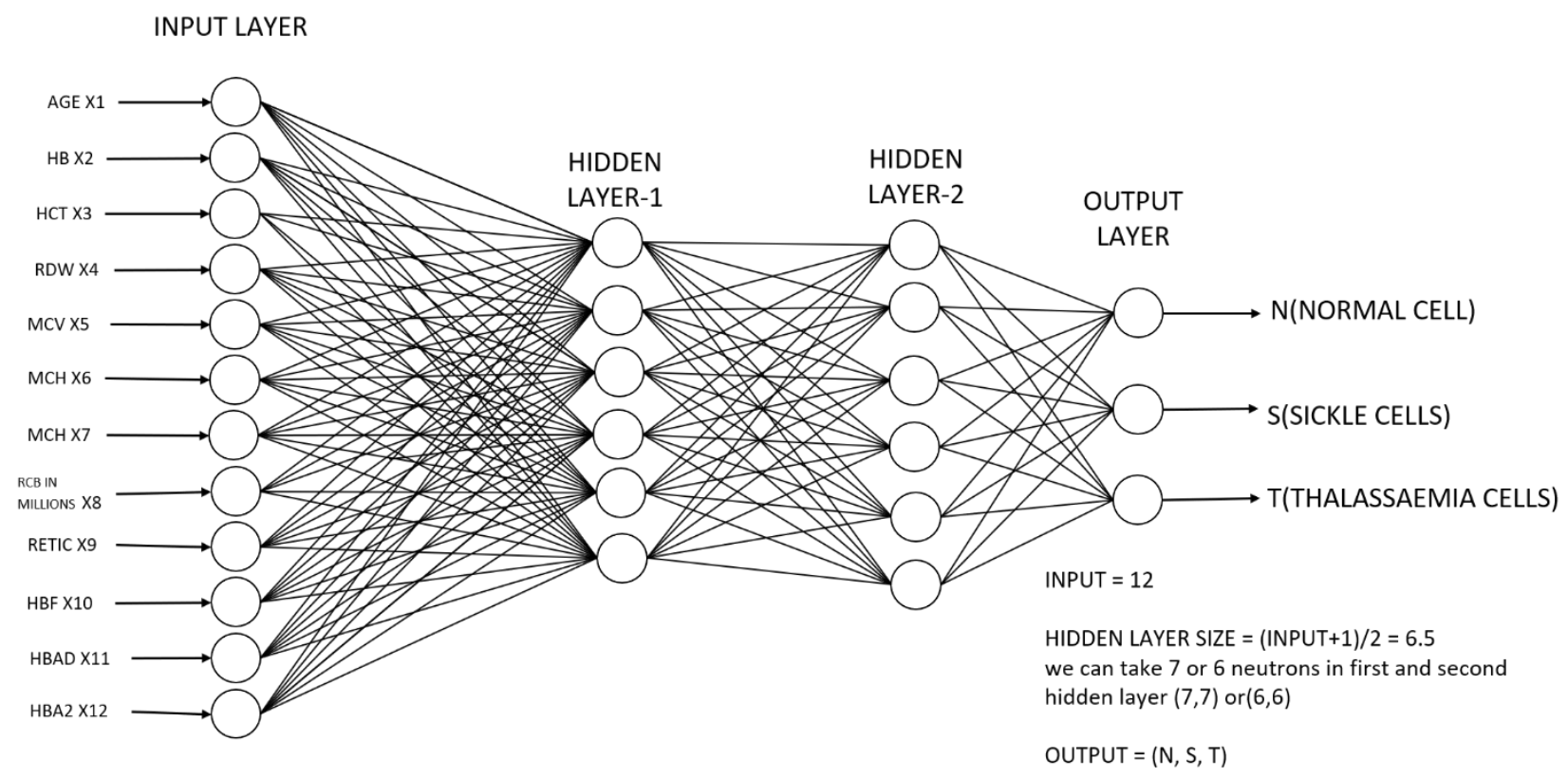

Figure 2. Architecture of the proposed network model for MLP classification.

As shown in the above figure-2, the proposed model of MLP comprises four layers of non-linearly triggering nodes (an input and an output layer of two hidden layers). Since MLPs are completely connected, each node in one-layer binds to each node in the corresponding layer, with a certain weight.

Training happens in the perceptron after analyzing each piece of data by adjusting relation weights, depending on the magnitude of the performance error compared with the expected result. It is an example of controlled learning and a generalization of the lowest mean square technique in linear vision by backpropagation.

MLP Classifier trains iteratively because the partial loss function derivatives are computed for updating the parameters in conjunction with model parameters in any step. It may also be applied to the loss function with a regularization time that reduces configuration parameters to avoid overfitting [31, 32]. 


\section{Confusion Matrix:}

\begin{tabular}{|c|c|}
\hline [[95 & 0 \\
\hline [1 & 39 \\
\hline [6 & 1 \\
\hline
\end{tabular}

Accuracy Score: 0.960431654676259

Report:

$\begin{array}{ccccc} & \text { Precision } & \text { recall } & \text { f1-Score } & \text { Support } \\ 0 & 0.93 & 0.97 & 0.95 & 98 \\ 1 & 0.97 & 0.97 & 0.97 & 40 \\ 2 & 0.98 & 0.95 & 0.96 & 140 \\ \text { Accuracy } & & & 0.96 & 278 \\ \text { Macro avg } & 0.96 & 0.96 & 0.96 & 278 \\ \text { Weighted avg } 0.96 & 0.96 & 0.96 & 278\end{array}$

Figure 3. Confusion Matrix of MLP Classifier.

\section{5- Results}

The classifier is implemented in PYTHON 3.6 with the configuration of the PC that has a processor Intel I7 running on Windows 10 OS with 16 GB RAM. The system suggested in this paper is measured based on confusion matrix, which includes Accuracy score, precision, recall, f1-score. Metrics assessment techniques [24] are defined as below fig-4:

\begin{tabular}{rlccc}
\multirow{2}{*}{ Confusion Matrix } & \multicolumn{3}{c}{ Predicted } \\
\cline { 2 - 5 } & Class 1 & Class 2 & Class 3 \\
\hline \multirow{3}{*}{ Actual } & Class 1 & A & B & C \\
\cline { 2 - 5 } & Class 2 & D & E & F \\
\cline { 2 - 5 } & Class 3 & G & H & I \\
\hline
\end{tabular}

True positives $\square$ True Negatives $\square$ Misclassified cases.

Furthermore, a confusion matrix is a table that reports the counts of true positive, false positive, true negatives and false negatives which are defined as:

- True Positive (TP): The label belongs to the class, and it is correctly predicted;

- False Positive (FP): The label does not belong to the class, but classifier predicted as positive;

- True Negative (TN): The label does not belong to the class, and it is correctly predicted;

- False Negative (FN): The label does not belong to the class, but is predicted as negative;

Figure 4. Metrics assessment techniques.

The following formulae is used in arriving the various measures of accuracy.

$$
\begin{aligned}
& \text { Accuracy }=\frac{T P+T N}{T P+T N+F P+F N} \\
& \text { Precision }=\frac{T P}{T P+F P} \\
& \text { Recall }=\frac{T P}{T P+F N} \\
& F-\text { Measure }=\frac{2 \times \text { Recall } \times \text { Precision }}{\text { Recall }+ \text { Precision }}
\end{aligned}
$$

And finally, the results obtained using all classification algorithms including the MLP is shown in the following Table 3. 
Table 3. Comparison study of various classification algorithms over the dataset including MLP classifier.

\begin{tabular}{cccc}
\hline S. No. & Name of the Classification Algorithm & $\begin{array}{c}\text { Result of prediction } \\
\text { accuracy (\%) }\end{array}$ & $\begin{array}{c}\text { Dataset Size (No of observations X } \\
\text { No of Parameters) }\end{array}$ \\
\hline 1 & Support Vector Machine (SVM) & 76.00 & 1387 X 13 \\
2 & K-Nearest Neighbor Algorithm (KNN) & 77.00 & 1387 X 13 \\
3 & Logistic Regression (L) & 78.00 & 1387 X 13 \\
4 & Decision Tree Classifier (DTC) & 95.00 & 1387 X 13 \\
5 & Random Forest Algorithm (RF) & 96.00 & 1387 X 13 \\
6 & MLP Classifier & 96.04 & 1387 X 13 \\
\hline
\end{tabular}

Validation of results: Sincere thanks to TSCS for providing an additional dataset of 100 patients records (data of patients approached to TSCS during September 2019 to August 2020) is selected for validation of the performance of the algorithms and is expressed as follows mentioned in Table 4.

Table 4. Validation results on dataset by various classification algorithms.

\begin{tabular}{cccc}
\hline S. No. & Name of the Classification Algorithm & $\begin{array}{c}\text { Result of prediction } \\
\text { accuracy (\%) }\end{array}$ & $\begin{array}{c}\text { Dataset Size (No of observations X } \\
\text { No of Parameters) }\end{array}$ \\
\hline 1 & Support Vector Machine (SVM) & 80.00 & 100 X 12 \\
2 & K-Nearest Neighbor Algorithm (KNN) & 83.00 & 100 X 12 \\
3 & Logistic Regression (L) & 82.00 & $100 \times 12$ \\
4 & Decision Tree Classifier (DTC) & 95.00 & $100 \times 12$ \\
5 & Random Forest Algorithm (RF) & 97.00 & 100 X 12 \\
6 & MLP Classifier & 99.00 & $100 \times 12$ \\
\hline
\end{tabular}

\section{5-1-Web Application Development}

A web application is developed based on the inputs given by TSCS especially that uses popular machine learning algorithms. The results are demonstrated to the team at TSCS using the Google Meet service. The team at TSCS has expressed their satisfaction and happiness about the performance of the application. The application is ready to deploy at the premises of TSCS for their regular usage.

\section{6- Conclusion}

Sickle cell anemia is a hematological condition, it was historically one of the peculiar traits of the indigenous community, but now it extends to the entire world and requires emergency treatment. This paper describes Sickle Cell Disease (SCD) and its history both internationally and in the national scenario (in the Indian context). The disease symptoms, signs, complications, and treatment are presented. Blood cells have also been characterized by different blood criteria among people with sickle-cell and Thalassemia patients. This paper further describes sickle cell disease with better precision with Multi-Layer Perceptron and builds an effective predictive model to minimize time and actions of sickle cell disease pain control systems. This paper examines the working model of the deep-learning model called "MLP Classifier" that gives efficient results than the other models like Support Vector Machine, K-Nearest Neighbor, Logistic Regression, Decision Tree Classifier and Random Forest Algorithms. The results of the simulations demonstrate that the proposed MLP classification has a 99 percent accuracy of prediction for both sickle cell and thalassemia. A web application is also developed for usage in the TSCS environment that includes the prediction model with the MLP classification algorithm even by a normal medical laboratory staff in an easier and simpler way.

\section{7- Declarations}

\section{7-1-Author Contributions}

Sagar Yeruva: Acts as the single point of contact for this project and the Principal Investigator. He is actively involved in all aspects of the project like communication to the funding agency like TEQIP-III of JNTU Hyderabad and to the MoU agency called TSCS. Sharada Varalakshmi: Acts as the Co-Principal Investigator and actively participates in all activities of the project and reporting to the funding agency. Pavan Gowtham. Bathula and Hari Chandana Y: Are the interns for this project who actively participated in collecting the literature survey and in execution of the project. These are also the students of M.Tech (Software Engineering) and B.Tech (Computer Science and Engineering) respectively in the department of Computer Science and Engineering in VNRVJIET, Hyderabad, Telangana, India. Krishna Prasad PESN: Acts as mentor for the project and supports in all activities of the project especially in executing the project environment. 


\section{7-2-Data Availability Statement}

The data collected as part of the project is from TSCS (Thalassemia and Sickle Cell Society, Hyderabad, India) which is a NGO dedicated for the services to Thalassemia and Sickle Cell affected people. The data is collected and shared with the project team as part of the MoU.

\section{7-3- Funding and Acknowledgements}

\section{7-3-1- JNTUH, TEQIP-III}

Sincere acknowledgements and thanks to Jawaharlal Nehru Technological University, Hyderabad, TEQIP-III (Technical Education Quality Improvement Programme-Phase III), for award of this research proposal under Collaborative Research Scheme, Proceedings No: JNTUH / TEQIP-III / CRS / 2019 / CSE / 04 dated 22-Jul-2019 for INR 3,00,000. Dr. Sagar Yeruva, acts as Principal Investigator and Dr. M. Sharada Varalakshmi acts as Co-Principal Investigator for this project. The duration of the project is from August-2019 to January-2021.

\section{7-3-2- Thalassemia and Sickle Cell Society (TSCS)}

Thalassemia and Sickle Cell Society, a NGO located in Rajendra Nagar, Hyderabad, Telangana, India which is a NGO working for the welfare of patients of Thalassemia and Sickle Cell blood disorders. Thanks to Dr. Suman Jain, CEO, TSCS who have accepted and executed the MoU. Sincere acknowledgements to the team at TSCS like Mr. Chandrakant Agarwal, President-TSCS, Dr. Suman Jain, Chief Medical Research Officer and Secretary-TSCS, Mr. Allam Ravi Kumar Reddy, Data Manager-TSCS, Dr. Saroja Kondaveeti, Medical Officer-TSCS, Dr. Padma Gunda, Research Scientist-TSCS, Mr. Mohd Abdul Tufeeq Baig, Lab Incharge, TSCS, Mr. Bhargava Kalvakota, Data \& Admin Officer-TSCS and Ch. Devasri, Data Entry Operator-TSCS who have helped us to understand the entire scenario of sickle cell patients, the process of their work and their services to the society in the state of Telangana, India.

\section{7-3-3- VNRVJIET}

Sincere acknowledgements to the management at VNRVJIET (Vallurupalli Nageswara Rao Vignana Jyothi Institute of Engineering \& Technology), Hyderabad especially, Dr. C. D. Naidu, Principal, Dr. B. Chennakeshava Rao, DeanAdministration, Dr. B. V. Kiranmayee, Head, Department of CSE, faculty and staff, Department of CSE for helping throughout the journey of this project and make it successful.

\section{7-4- Conflicts of Interest}

The author declares that there is no conflict of interests regarding the publication of this manuscript. In addition, the ethical issues, including plagiarism, informed consent, misconduct, data fabrication and/or falsification, double publication and/or submission, and redundancies have been completely observed by the authors.

\section{8- References}

[1] Westerman, Maxwell, and John B. Porter. "Red Blood Cell-Derived Microparticles: An Overview." Blood Cells, Molecules, and Diseases 59 (July 2016): 134-139. doi:10.1016/j.bcmd.2016.04.003.

[2] Paula Tanabe, "Sickle Cell Disease core concepts for emergency physician and nurse", Available online: https://slideplayer.com/slide/3762536/ (accessed on February 2021).

[3] Stuart, Marie J, and Ronald L Nagel. "Sickle-Cell Disease." The Lancet 364, no. 9442 (October 2004): 1343-1360. doi:10.1016/s0140-6736(04)17192-4.

[4] Sickle cell anemia. Available online: https://www.mayoclinic.org/diseases-conditions/sickle-cell-anemia/symptoms causes/syc20355876 (accessed on February 2021).

[5] Serjeant, Graham R. "Sickle-Cell Disease.” The Lancet 350, no. 9079 (September 1997): 725-730. doi:10.1016/s01406736(97)07330-3.

[6] Sickle Cell Disease. Available online: https://www.nhlbi.nih.gov/health-topics/sickle-cell-disease (accessed on January 2021).

[7] Yawn, Barbara P., George R. Buchanan, Araba N. Afenyi-Annan, Samir K. Ballas, Kathryn L. Hassell, Andra H. James, Lanetta Jordan, et al. "Management of Sickle Cell Disease." JAMA 312, no. 10 (September 10, 2014 ): 1033. doi:10.1001/jama.2014.10517.

[8] Chy, Tajkia Saima, and Mohammad Anisur Rahaman. "A Comparative Analysis by KNN, SVM \& ELM Classification to Detect Sickle Cell Anemia." 2019 International Conference on Robotics, Electrical and Signal Processing Techniques (ICREST) (January 2019). doi:10.1109/icrest.2019.8644410.

[9] Data \& Statistics on Sickle Cell Disease. Available online: https://www.cdc.gov/ncbddd/sicklecell/data.html (accessed on January 2021). 
[10] Platt, Orah S., Bruce D. Thorington, Donald J. Brambilla, Paul F. Milner, Wendell F. Rosse, Elliott Vichinsky, and Thomas R. Kinney. "Pain in Sickle Cell Disease." New England Journal of Medicine 325, no. 1 (July 4, 1991): 11-16. doi:10.1056/nejm199107043250103.

[11] Vichinsky, Elliott P., Lynne D. Neumayr, Ann N. Earles, Roger Williams, Evelyne T. Lennette, Deborah Dean, Bruce Nickerson, et al. "Causes and Outcomes of the Acute Chest Syndrome in Sickle Cell Disease." New England Journal of Medicine 342, no. 25 (June 22, 2000): 1855-1865. doi:10.1056/nejm200006223422502.

[12] Elsalamony, Hany A. "Healthy and Unhealthy Red Blood Cell Detection in Human Blood Smears Using Neural Networks." Micron 83 (April 2016): 32-41. doi:10.1016/j.micron.2016.01.008.

[13] Salman, Muhammad, Amer Hayat Khan, Azreen Syazril Adnan, Syed Azhar Syed Sulaiman, Khalid Hussain, Naureen Shehzadi, Muhammad Islam, and Fauziah Jummaat. "Prevalence and Management of Anemia in Pre-Dialysis Malaysian Patients: A Hospital-Based Study.” Revista Da Associação Médica Brasileira 62, no. 8 (November 2016): 742-747. doi:10.1590/18069282.62.08.742.

[14] Vichinsky, Elliott, Mel Heyman, Deborah Hurst, Danny Chiu, Barbara Gaffield, Karen Thompson, Klara Kleman, and Bertram Lubin. "NUTRITION IN SICKLE CELL ANEMIA (HB SS)." Pediatric Research 18 (April 1984): 251A-251A. doi:10.1203/00006450-198404001-00946.

[15] Pennap, Grace, and Khadijah Abubakar. "Prevalence of Anemia Among Human Immunodeficiency Virus Infected Patients Accessing Healthcare in Federal Medical Center Keffi, Nigeria." International Journal of TROPICAL DISEASE \& Health 10, no. 3 (January 10, 2015): 1-7. doi:10.9734/ijtdh/2015/19657.

[16] Yeruva, Sagar, M. Sharada Varalakshmi, B. Pavan Gowtham, Y. Hari Chandana, and P. E. S. N. Krishna Prasad. "Sickle Cell Disease - A Comprehensive Study and Usage of Technology for Diagnosis.” International Blood Research \& Reviews (June 19, 2020): 6-14. doi:10.9734/ibrr/2020/v11i230125.

[17] Solanki, Ashokkumar Vijaysinh. "Data mining techniques using WEKA classification for sickle cell disease." International Journal of Computer Science and Information Technologies 5, no. 4 (2014): 5857-5860.

[18] Chadha, Gulpreet Kaur, Aakarsh Srivastava, Abhilasha Singh, Ritu Gupta, and Deepanshi Singla. "An Automated Method for Counting Red Blood Cells Using Image Processing.” Procedia Computer Science 167 (2020): 769-778. doi:10.1016/j.procs.2020.03.408.

[19] Ilyas, Shazia, Andrew Evan Simonson, and Waseem Asghar. "Emerging Point-of-Care Technologies for Sickle Cell Disease Diagnostics.” Clinica Chimica Acta 501 (February 2020): 85-91. doi:10.1016/j.cca.2019.10.025.

[20] Hortinela, Carlos C., Jessie R. Balbin, Janette C. Fausto, Paul Daniel C.Divina, and John Philip T. Felices. "Identification of Abnormal Red Blood Cells and Diagnosing Specific Types of Anemia Using Image Processing and Support Vector Machine." 2019 IEEE 11th International Conference on Humanoid, Nanotechnology, Information Technology, Communication and Control, Environment, and Management (HNICEM) (November 2019). doi:10.1109/hnicem48295.2019.9072904.

[21] Alzubaidi, Laith, Omran Al-Shamma, Mohammed A. Fadhel, Laith Farhan, and Jinglan Zhang. "Classification of Red Blood Cells in Sickle Cell Anemia Using Deep Convolutional Neural Network.” Intelligent Systems Design and Applications (April 12, 2019): 550-559. doi:10.1007/978-3-030-16657-1_51.

[22] Elsalamony, Hany A. "Detection of Anaemia Disease in Human Red Blood Cells Using Cell Signature, Neural Networks and SVM." Multimedia Tools and Applications 77, no. 12 (August 19, 2017): 15047-15074. doi:10.1007/s11042-017-5088-9.

[23] Xu, Mengjia, Dimitrios P. Papageorgiou, Sabia Z. Abidi, Ming Dao, Hong Zhao, and George Em Karniadakis. “A Deep Convolutional Neural Network for Classification of Red Blood Cells in Sickle Cell Anemia." Edited by Qing Nie. PLOS Computational Biology 13, no. 10 (October 19, 2017): e1005746. doi:10.1371/journal.pcbi.1005746.

[24] Aruna, N. S., and S. Hariharan. "A Level-Set-Based Segmentation for the Detection of Megaloblastic Anemia in Red Blood Cells.” Lecture Notes in Networks and Systems (October 24, 2017): 119-128. doi:10.1007/978-981-10-5523-2_12.

[25] Savkare, S. S., A. S. Narote, and S. P. Narote. "Automatic Blood Cell Segmentation Using K-Mean Clustering from Microscopic Thin Blood Images." Proceedings of the Third International Symposium on Computer Vision and the Internet (September 21, 2016). doi:10.1145/2983402.2983409.

[26] Khalaf, Mohammed, Abir Jaafar Hussain, Robert Keight, Dhiya Al-Jumeily, Paul Fergus, Russell Keenan, and Posco Tso. "Machine Learning Approaches to the Application of Disease Modifying Therapy for Sickle Cell Using Classification Models." Neurocomputing 228 (March 2017): 154-164. doi:10.1016/j.neucom.2016.10.043.

[27] Gowtham, Bathula Pavan, Yendluri Hari Chandana, Sagar Yeruva, M. Sharada Varalakshmi, PESN Krishna Prasad, Suman Jain, Allam Ravi Kumar Reddy, Saroja Kondaveeti, and Padma Gunda. Prediction of Anemia Disease Using Classification Methods. No. 3164. EasyChair, (2020).

[28] Sanap, Shilpa A., Meghana Nagori, and Vivek Kshirsagar. "Classification of Anemia Using Data Mining Techniques.” Lecture Notes in Computer Science (2011): 113-121. doi:10.1007/978-3-642-27242-4_14. 
[29] Alzubaidi, Laith, Mohammed A. Fadhel, Omran Al-Shamma, Jinglan Zhang, and Ye Duan. "Deep Learning Models for Classification of Red Blood Cells in Microscopy Images to Aid in Sickle Cell Anemia Diagnosis." Electronics 9, no. 3 (March 4, 2020): 427. doi:10.3390/electronics9030427.

[30] Understanding of Multilayer perceptron (MLP). Available online: https://medium.com/@AI_with_Kain/understanding-ofmultilayer-perceptron-mlp-8f179c4a135f (accessed on February 2021).

[31] Parvez, Mohammad K., and Sakina Niyazi. "The Genomic and Structural Organization of SARS-CoV-2: A Mutational Perspective." SciMedicine Journal 3, no. 1 (March 1, 2021): 59-65. doi:10.28991/scimedj-2021-0301-8.

[32] Meena, Kanak, Devendra K. Tayal, Vaidehi Gupta, and Aiman Fatima. "Using Classification Techniques for Statistical Analysis of Anemia.” Artificial Intelligence in Medicine 94 (March 2019): 138-152. doi:10.1016/j.artmed.2019.02.005. 